\title{
Unsteady dynamics in the streamwise-oscillating cylinder wake for forcing frequencies below lock-on
}

\author{
Maysam Shamai $\odot^{*}$ \\ Graduate Aerospace Laboratories, California Institute of Technology, Pasadena, California 91125, USA \\ Scott T. M. Dawson 10 \\ Department of Mechanical, Materials, and Aerospace Engineering, \\ Illinois Institute of Technology, Chicago, Illinois 60616, USA \\ Igor Mezić \\ Department of Mechanical Engineering, University of California Santa Barbara, Santa Barbara, \\ California 93106, USA \\ Beverley J. McKeon (1) \\ Graduate Aerospace Laboratories, California Institute of Technology, Pasadena, California 91125, USA
}

(Received 10 July 2020; accepted 1 April 2021; published 12 July 2021)

\begin{abstract}
The flow around a cylinder oscillating in the streamwise direction with a frequency, $f_{f}$, much lower than the shedding frequency, $f_{s}$, has been relatively less studied than the case when these frequencies have the same order of magnitude or the transverse oscillation configuration. In this study, particle image velocimetry and the dynamic mode decomposition (DMD) are used to investigate the streamwise-oscillating cylinder wake for forcing frequencies $f_{f} / f_{s} \sim 0.04-0.2$ and mean Reynolds number, $\operatorname{Re}_{0}=900$. The amplitude of oscillation is such that the instantaneous Reynolds number remains above the critical value for vortex shedding at all times. Characterization of the wake reveals a range of phenomena associated with the interaction of the two frequencies, including modulation of both the amplitude and frequency of the wake structure by the forcing. DMD reveals a frequency spreading of dynamic modes. A scaling parameter and associated transformation are developed to relate the unsteady, or forced, dynamics of a system to that of an unforced system. For the streamwise-oscillating cylinder, it is shown that this transformation leads to a dynamic mode decomposition similar to that of the unforced system.
\end{abstract}

DOI: 10.1103/PhysRevFluids.6.074702

\section{INTRODUCTION}

Fluid-structure interaction serves as the backdrop of many important problems involving flow physics. Even the presence of a simple structure in the flow leads to additional boundary conditions that can significantly alter the system's dynamics. The complexity of the system is further increased when the structure takes on motion, whether predefined or in the form of a response to the flow. Although a moving structure introduces an added level of complexity, often many of the flow structures encountered may also be seen for the fixed case. This motivates the desire to explain and/or approximate the dynamics of the moving system using only knowledge of its fixed counterpart. The

*mshamai@caltech.edu 
difficulty, however, lies in determining which characteristics of the flow carry over and, if so, are the underlying physics governing them related. In this work, the streamwise-oscillating cylinder is used to study this question and develop a framework to relate the moving and fixed fluid-structure problems. Specifically, theory, experiment, and reduced-order modeling are used to address various aspects of the problem.

An expansive body of work considers the flow around oscillating cylinders; examples include Williamson and Roshko [1] and Williamson and Govardhan [2]. Examining the literature, however, reveals that only a small subset of studies consider streamwise oscillations. This is primarily due to significant interest in transverse oscillations and their role in vortex-induced vibration. Streamwise forcing induces relatively stronger changes in the instantaneous Reynolds number, adding interest to the problem as well as delineating it from the transverse case. Hence, understanding the dynamics of the streamwise-oscillating cylinder could prove useful in categorizing structures seen in the wake of an unsteady body into those generated by changes in the Reynolds number and those specific to the body's geometry. Within the body of work considering streamwise oscillations, the majority of studies consider forcing frequencies, $f_{f}$, of the same order of magnitude (or higher) than the stationary shedding frequency, $f_{0}$. This regime is of interest due to the lock-on phenomenonthe synchronization between the shedding frequency, $f_{s}$, and forcing frequency, $f_{f}$-that occurs for certain frequency ratios when the streamwise forcing frequency is approximately equal to the stationary shedding frequency [3].

The phenomena that arise due to streamwise forcing are intricately related to vortex dynamics as well as the forcing frequency and amplitude. Leontini et al. [4] studied the streamwise-oscillating cylinder using two-dimensional computations with mean Reynolds number, $\operatorname{Re}_{0}=175$. They considered a forcing frequency equivalent to the stationary shedding frequency and deduced that the development of frequency modulation in the wake is governed by the forcing frequency while vortex interactions lead to the development of amplitude modulation. Leontini et al. [5] parametrically studied the streamwise-oscillating cylinder for $75 \leqslant \mathrm{Re}_{0} \leqslant 250$ and forcing frequencies in the range $1 \leqslant f_{f} / f_{0} \leqslant 2$.

Konstantinidis et al. [6] studied a cylinder allowed to oscillate in-line with the flow subject to a steady freestream. In an effort to model the force on the cylinder, they conducted a parametric study of the system at low Reynolds numbers. They presented the development of a resonant oscillation mode corresponding to the synchronization between shedding and body motion. Even though both the shedding and oscillation were synchronized, it was seen that the relative phase between the two played an important role in determining the force felt by the cylinder. A deeper understanding of the phase-dependent dynamics plays a vital role in understanding and modeling the system.

Glaz et al. [7] used Koopman analysis to analyze the temporal behavior of a streamwiseoscillating cylinder with mean Reynolds number, $\mathrm{Re}_{0}=53$ (close to the critical value). They considered a forcing frequency two orders of magnitude less than the stationary shedding frequency for various oscillation amplitudes. Koopman analysis was performed in parallel to two-dimensional flow simulations and the results were in good agreement. Glaz et al. showed that the flow in this regime was marked by instances of strong vortex shedding which were then replaced by periods of suppressed shedding and weak oscillatory behavior, a phenomenon they refer to as "quasiperiodic intermittency." Additionally, they observed that streamwise forcing led to the extraction of additional flow structures (modes) related to vortex shedding as well as a broadening of the associated spectral peak.

The current study examines forcing frequencies one and two orders of magnitude less than the stationary shedding frequency. It is has previously been shown that streamwise forcing not corresponding to lock-on can lead to wake patterns that significantly deviate from the classic Kármán vortex street [3-5,8]. The effect of forcing frequencies one-to-two orders of magnitude smaller than the stationary shedding frequency has received considerably less attention than the lock-on case, even though it corresponds to numerous fluid-structure systems. For example, this frequency ratio corresponds approximately to vortex shedding from an airfoil undergoing dynamic stall or the development of a wake breathing mode. A body in the wake of another can also be 
subject to an oncoming flow characterized by a similar frequency ratio, namely, $f_{f} \ll f_{0}$, with direct importance for the modeling of the disturbance environment and potential energy harvesting applications. Numerous flow structures present in the streamwise-oscillating cylinder wake also appear in other fluid-structure systems.

Modal decompositions have proven useful in both the extraction of dynamically significant flow structures as well as reduced-order modeling $[9,10]$. In this study, the dynamic mode decomposition (DMD) is used to extract dynamically significant flow structures. The dynamic mode decomposition specifically refers to the eigendecomposition of the linear operator that best approximates system dynamics [11]. The power of DMD lies in its ability to extract flow structures that are of spatiotemporal importance [11,12]. One advantage of DMD is its ability to extract the temporal growth rate of spatial structures (modes) [13]. Many spectral analysis techniques, such as the temporal discrete Fourier transform (DFT), only yield mode frequencies and not growth rates, limiting the insight they give into systems with high degrees of unsteadiness or transient behavior. Also, in contrast to the temporal DFT, the set of frequencies extracted by DMD does not have to be equally spaced [13], making DMD a good candidate for studying systems with dynamics distributed over a range of unequally spaced frequencies. DMD also has connections to the Koopman operator, an infinite-dimensional linear operator used to study the dynamics of nonlinear systems [12]. These connections will only be discussed briefly here, but readers can refer to Refs. [12,14] for more detailed discussion.

DMD has been used extensively to study a wide range of systems [9], specifically to identify dominant linear dynamics and to inform reduced complexity flow models. It will be shown that basic DMD of the streamwise-oscillating cylinder's wake does not lead to an efficient representation of the flow. However, it will be shown that application of a quasisteady scaling, leads to DMD results that can more clearly be related to the system dynamics. The quasisteady timescaling, combined with the fact that DMD appeals to the best-fit linear operator approximating system dynamics, motivates a path forward in future work to create a reduced-order model of the system which can be used to accurately reconstruct the flow.

Although the use of quasisteady approximations is not unique to fluid mechanics, the underlying theme is unifying. That is, a system exhibiting activity spanning multiple timescales can be simplified greatly if the timescales are sufficiently separated such that the dynamics at one scale can be considered steady relative to the others $[15,16]$. This assumption has proven invaluable in the understanding and modeling of fluid flows. One example includes the work of Sane and Dickinson [17], who used a quasisteady model to characterize different phenomena contributing to the force exerted on a flapping wing. One objective of the current study is the development of a framework that can be used to identify forcing trajectories where the assumption of quasisteady dynamics is appropriate for the streamwise-oscillating cylinder. In this analysis, quasisteady references behavior similar to the stationary, or unforced, case, i.e., periodic vortex shedding in the wake. In physical terms, the corresponding question is whether the timescale of the forcing is sufficiently slow relative to the time taken to shed a vortex such that the slow scale dynamics can be neglected on a vortex by vortex basis.

The remainder of this paper is organized as follows. In Sec. II, the experimental approach, namely particle image velocimetry, used to study the flow around the streamwise-oscillating cylinder is outlined. The framework used to compute dynamic modes and eigenvalues from experimental velocity fields is also discussed, and the scaling to transform the dynamics of the unsteady system to one exhibiting quasisteady dynamics is given. The results of oscillating-cylinder experiments, the associated DMD, and timescaling is presented in Sec. III. In Sec. IV results are discussed in more detail and the implications of scaling on the data are highlighted. Finally, concluding remarks are given Sec. V. 


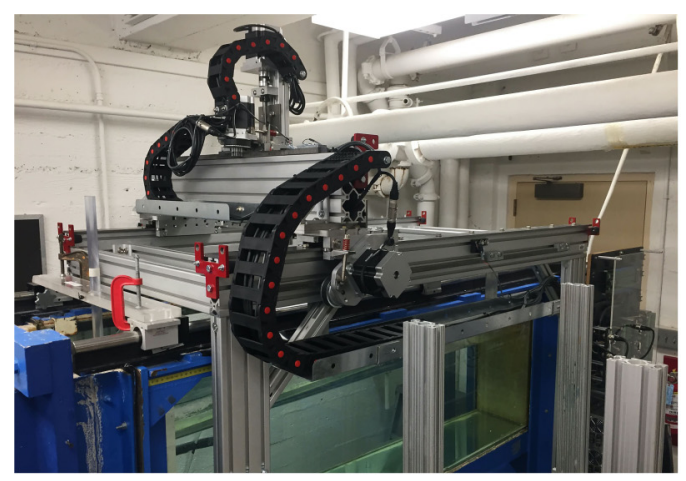

(a)

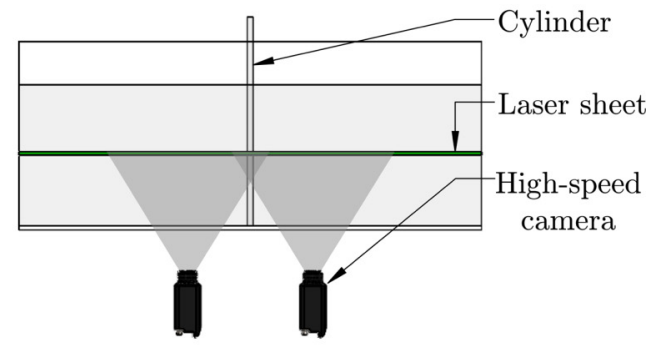

(b)

FIG. 1. (a) The captive trajectory system mounted on top of the NOAH water channel's test section. Flow is from left to right. (b) Simplified diagram of experimental setup.

\section{METHODS}

\section{A. Oscillating cylinder experiments}

In order to study the flow around a streamwise-oscillating cylinder, experiments were carried out in the NOAH free-surface water channel at Caltech, a recirculating facility shown in Fig. 1(a). A captive trajectory system (CTS) allows for translation of a model along all three coordinate axes as well as rotation about two of those axes. The CTS spans the length of the test section, approximately $2 \mathrm{~m}$ in length, and can move with submillimeter accuracy reaching speeds up to $1 \mathrm{~m} / \mathrm{s}$. In this study a prescribed trajectory is used to generate streamwise oscillations.

An acrylic cylinder with a diameter of $19 \mathrm{~mm}$ and wetted length of approximately half a meter was mounted on the CTS such that its axis pointed downwards towards the bottom of the test section. In order to reduce end effects, the bottom of the cylinder was located approximately one millimeter from the bottom of the test section.

The range of Reynolds numbers considered here $\left(\operatorname{Re}_{0} \sim 10^{3}\right)$ can lead to three-dimensional structures in the wake, including, but not limited to, vortex dislocations and oblique shedding angles [18]. The present study primarily focuses on dominant, large-scale structures in the wake, such as shed vortices. It will be shown that these structures are clearly distinguishable in two-dimensional flow fields. In fact, consideration of the transverse component of velocity $(v)$ alone gives significant insight into the wake dynamics. As such, two-dimensional particle image velocimetry PIV was the primary diagnostic used to acquire velocity fields. A Photonics DM20-527(nm) dual-head YLF laser was pulsed to illuminate tracer particles. Images of the illuminated particles were acquired using two Phantom Miro Lab 320 cameras with AF Nikkor 50-mm $f / 1$.8D lenses. The cameras were situated under the test section and normal to the laser sheet, as seen in Fig. 1(b). The laser and optics were configured such that the laser sheet was concurrent with the cross section of the cylinder. Experiments were performed using both single-pulse and double-pulse laser configurations. Singlepulse experiments produced a higher time resolution but shorter record length while double pulse experiments lead to a longer record length but reduced time resolution. The sampling rate for single pulse experiments was $70 \mathrm{~Hz}$ while double pulse experiments had an effective sampling rate of $12 \mathrm{~Hz}$. The LaVision DaVis PIV software suite was used for calibration, image acquisition, and postprocessing. The PIV algorithm used to compute velocity fields consisted of three passes with the final iteration using a 24 -by-24 pixel window with $50 \%$ overlap. Finally, a 3-by-3 smoothing filter was applied to reduce noise in the flow fields.

Besides the full-field information, the velocity from an interrogation point located four diameters downstream of the cylinder will also be discussed in order to compare local and global dynamics. 
The interrogation point was not a separate measurement or probe in the wake but rather a single point in the PIV flow fields from which the velocity was extracted. To maintain a constant distance from the cylinder, the interrogation point was translated in phase with the cylinder during the forcing trajectory. PIV was conducted in the laboratory frame leading to relative motion between the cylinder and camera. Translation of the interrogation point combined with finite PIV grid resolution lead to a small pixelation error in the interrogation point. That is, the interrogation point was chosen as the grid point closed to the desired distance downstream of the cylinder. The pixelation error was computed to be at most $1.5 \mathrm{~mm}$ and did not introduce noticeable artifacts in time traces of velocity at the interrogation point.

In order to compare the global DMD analysis to a local spectral analysis, Welch's method was used to compute the power spectral density (PSD) for the mean-subtracted, transverse component of velocity at the interrogation point. Specifically, Hamming windows with no overlap and a window size corresponding to half of the forcing cycle were used. Furthermore, each window was padded with zeros to provide a finer spectral resolution. This choice of window was used in order to identify the two dominant frequencies exhibited by the system, with one present in each half of the forcing cycle. Finite camera memory resulted in the recording of fewer cycles for cases corresponding to smaller forcing frequencies. This corresponded to the use of fewer windows in Welch's method and interrogation point spectra that might not be fully converged for lower frequency cases.

Many of the results discussed above are linked to certain phases in the forcing cycle. Phaseaveraging is employed to gain a deeper understanding of these phase-locked phenomena. Results from double-pulse experiments are used because they correspond to a longer record length and hence contain more forcing cycles. Phase-averaged results, presented in Sec. III B 2, incorporate 42 forcing cycles with each forcing cycle consisting of 129 snapshots. Phase-averaging is completed by separating each time series into individual forcing cycles then averaging across all the cycles (corresponding to the same forcing parameters).

In addition to PIV, fluorescent dye was used to visualize unsteady structures present in the flow. A dye injection port was located at the upstream stagnation point of the cylinder and (vertically) at the center of the test section. Fluorescent water tracing dye, produced by Ecoclean Solutions, was injected at the upstream stagnation point and illuminated using two HouLight 50-W ultraviolet floodlights. A thin slit was placed in front of the UV floodlights to reduce the beam width and increase contrast in images. The flow rate of the dye was adjusted to ensure no jet was produced (which would perturb the cylinder boundary layers); rather, a thin filament of dye advected evenly on both sides of the cylinder and into the wake. A Nikon D600 DSLR camera with an AF Nikkor $50-\mathrm{mm} f / 1.8 \mathrm{D}$ lens was used to record images of the illuminated dye. In contrast to PIV, where images were acquired in the laboratory-fixed frame, the DSLR was mounted onto the CTS facing down along the axis of the cylinder, i.e., images were acquired in the body-fixed frame.

\section{B. Cylinder trajectory}

The cylinder was translated in the streamwise direction with harmonic variation of streamwise position while the freestream velocity was held constant. Each cylinder trajectory consisted of a sinusoidal oscillation of streamwise position with angular frequency $\omega_{f}=2 \pi / \tau_{f}$. The freestream velocity, $U_{\infty}$, was held constant, such that the effective instantaneous velocity experienced by the cylinder is given by

$$
U(t)=U_{\infty}-\frac{2 q}{\omega_{f}} \sin \left(\omega_{f} t\right)
$$

The parameter $q$, with units of acceleration, characterizes the amplitude of forcing, after Glaz et al. [7] [note that the latter study was performed in a different frame of reference, namely, a stationary cylinder experienced $U_{\infty}(t)$ ]. The corresponding variation of instantaneous Reynolds number is as 
TABLE I. Forcing parameters considered in oscillating cylinder experiments.

\begin{tabular}{lccc}
\hline \hline $\mathrm{Re}_{0}$ & $\mathrm{Re}_{q}$ & $\mathrm{St}_{f} / \mathrm{st}_{0}$ & $\mathrm{Re}_{q} / \mathrm{Re}_{0}$ \\
\hline 900 & 16 & 0.018 & 0.018 \\
900 & 160 & 0.018 & 0.18 \\
900 & 16 & 0.036 & 0.018 \\
900 & 160 & 0.036 & 0.18 \\
900 & 320 & 0.036 & 0.35 \\
900 & 16 & 0.18 & 0.018 \\
900 & 160 & 0.18 & 0.18 \\
900 & 320 & 0.18 & 0.35 \\
\hline \hline
\end{tabular}

follows:

$$
\operatorname{Re}(t)=\frac{U(t) D}{v}=\operatorname{Re}_{0}-\operatorname{Re}_{q} \sin \left(\omega_{f} t\right) .
$$

Here $D$ and $v$ are the cylinder diameter and the kinematic viscosity of the fluid, respectively. $\operatorname{Re}_{0}=\frac{U_{\infty} D}{v}$ corresponds to the mean Reynolds number, while $\operatorname{Re}_{q}=\frac{2 D q}{v \omega_{f}}$ characterizes the forced perturbation around the mean. $\operatorname{Re}_{0}$ and $\operatorname{Re}_{q}$, combined with the forcing Strouhal number, $\mathrm{St}_{f}=\frac{\omega_{f} D}{2 \pi U}$, can be changed to modify the forcing trajectory. For flow over a stationary cylinder, the relation between the Strouhal number, $\mathrm{St}_{0}$, and the Reynolds number, $\mathrm{Re}_{0}$, is well characterized [19].

Experiments were performed for multiple combinations of forcing frequency and amplitude at a mean Reynolds number of $\operatorname{Re}_{0}=900$. Forcing frequencies both one and two orders of magnitude less the stationary shedding frequency were considered. Throughout the remainder of this paper, $\mathrm{St}_{f}<\mathrm{St}_{0}$ will refer to cases with a forcing frequency one order of magnitude less than the stationary shedding frequency while $\mathrm{St}_{f} \ll \mathrm{St}_{0}$ will designate cases with a two order of magnitude difference between forcing and stationary shedding frequencies. Table I presents a summary of the various combinations of forcing frequency and amplitude considered in experiments; the time-dependent Reynolds number experienced by the cylinder is shown in Fig. 2(a). In this study, experiments utilized forcing parameters that ensured the instantaneous Reynolds numbers remained above the critical value at all times. It should be noted that although the mean Reynolds number considered in this study varied from that considered by Glaz et al. [7], there was still some overlap in ratios of both forcing frequency and amplitude, $\frac{\mathrm{St}_{f}}{\mathrm{St}_{0}}$, and $\frac{\mathrm{Re}_{q}}{\mathrm{Re}_{0}}$, respectively.

Each forcing trajectory was such that the stationary shedding Strouhal number, St, corresponding to the instantaneous Reynolds number was approximately constant with a value of $\mathrm{St} \approx \mathrm{St}_{0}=0.21$. From the definition of Strouhal number, it can be seen that for constant St, any change in freestream velocity, $U$, must be balanced by a variation of the shedding frequency, $f$. Making a quasisteady assumption, namely, $\mathrm{St} \approx \mathrm{St}_{0}=$ const, allows the instantaneous shedding frequency to be expressed as

$$
f(t)=\frac{\mathrm{St}_{0} U(t)}{D}
$$

or for the harmonic variation of cylinder velocity given in Eq. (1),

$$
f(t)=\frac{\mathrm{St}_{0} v}{D^{2}}\left[\operatorname{Re}_{0}-\operatorname{Re}_{q} \sin \left(\omega_{f} t\right)\right] .
$$

\section{Identification of quasisteady forcing regimes}

Consider the effect of simple harmonic forcing with period $\tau_{f}$ on a system with a time varying characteristic shedding frequency and period, $f(t)$ and $\tau(t)=\frac{1}{f(t)}$, respectively. Examine first the rate of change of the characteristic frequency and period, $\dot{f}$ and $\dot{\tau}$, respectively. Although both 


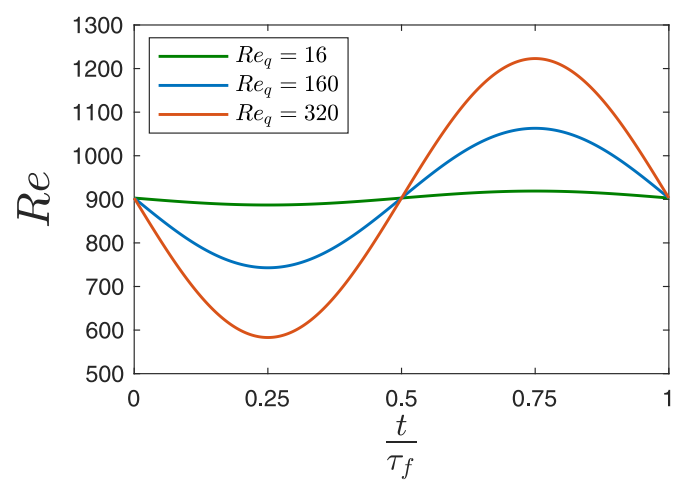

(a)

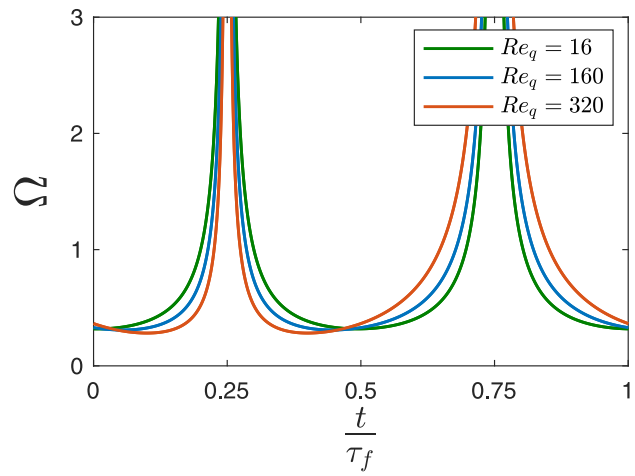

(c)

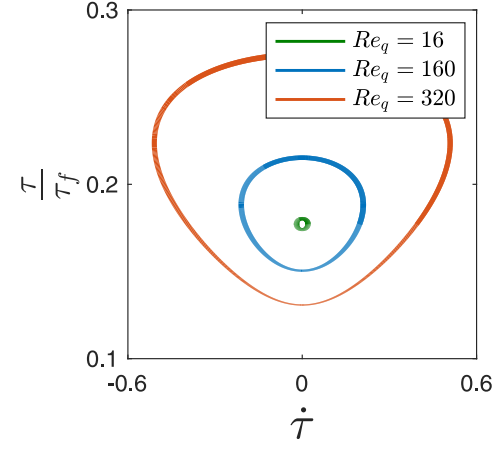

(b)

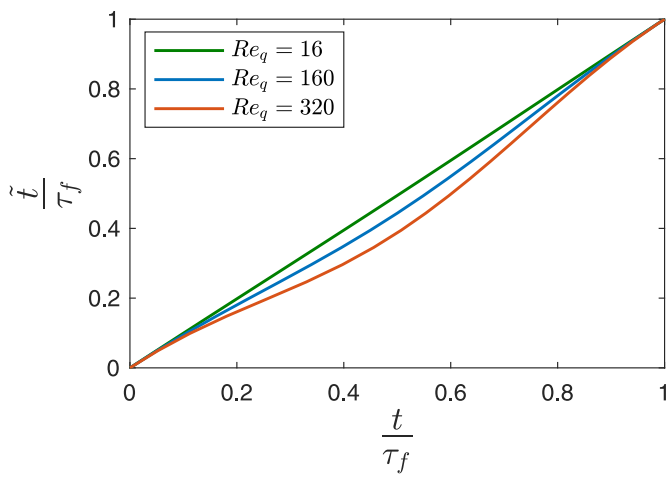

(d)

FIG. 2. (a) Instantaneous Reynolds number, $\operatorname{Re}(t)$, corresponding to experimental forcing trajectory. (b) Phase portrait showing change in $\tau$ and $\dot{\tau}$ during forcing cycle. Linewidth is proportional to speed in phase space. (c) Quasisteadiness parameter plotted for streamwise forcing trajectory. (d) Variation in scaled time, $\tilde{t}$, with laboratory time, $t$.

parameters serve as a proxy of the rate at which system dynamics are changing, they do not both convey the same level of information. It is important to note that

$$
\dot{\tau}=-\frac{\dot{f}}{f^{2}},
$$

whereas $\dot{f}$ is simply the instantaneous rate of change in frequency, $\dot{\tau}$ also incorporates the instantaneous frequency, $f$ (and thus will take different values during the upstream and downstream portions of the cylinder trajectory). This is particularly important for the oscillating cylinder as vortex shedding requires that both the shedding frequency and its rate of change be such that shedding can develop during the forcing cycle. Thus, $\dot{\tau}$ will primarily be considered in the following analysis. Normalizing $\dot{\tau}$ with the quantity

$$
\frac{\Delta \tau}{\tau_{f}},
$$

where $\Delta \tau=\tau_{\max }-\tau_{\min }$, the maximum change in characteristic period during the forcing cycle, scales $\dot{\tau}$ and leads to the parameter

$$
\Omega(t)=\left|\frac{\Delta \tau}{\tau_{f} \dot{\tau}}\right|,
$$


referred to as the "quasisteadiness" in the remainder of this paper. Since the dependence of frequency on time is known through Eq. (4), $\Omega$ can be computed a priori,

$$
\Omega=\left|\frac{\sec \left(\omega_{f} t\right)\left[\mathrm{Re}_{0}-\mathrm{Re}_{q} \sin \left(\omega_{f} t\right)\right]^{2}}{\pi\left(\mathrm{Re}_{0}^{2}-\mathrm{Re}_{q}^{2}\right)}\right| .
$$

From the Taylor series perspective, $\Omega$ gives a time-dependent measure of the closeness of the system to steady behavior in a linear sense. An alternate interpretation of $\left|\Delta \tau / \tau_{f} i\right|$ is as a normalized timescale associated with the change in shedding period. [In fact, Segel and Slemrod [15] use a similar expression, albeit with a chemical concentration instead of the characteristic period, $\tau$, to define the "slow" timescale in a quasisteady model of a chemical reaction.] The quasisteadiness parameter in this work has been formulated from the latter perspective. Consequently, $\Omega \gg 1$ indicates that the temporal behavior of the system is evolving relatively slowly and hence the system may be quasisteady. It is important to note that large values of $\Omega$ do not necessarily guarantee quasisteady dynamics, especially in cases where the separation of scales between the characteristic and forcing frequency is not large (i.e., if there is not enough time to develop consistent vortex shedding during the forcing cycle). Rather, high values of $\Omega$ allude to portions of the forcing cycle most likely to exhibit quasisteady behavior and vice versa.

The quasisteady assumption implies that the system dynamics are such that the fundamental mechanism of vortex shedding remains the same, subject only to an adjustment in frequency in response to the change in the effective instantaneous Reynolds number. Figure 2(b) shows the phase portrait relating $\dot{\tau}$ and $\tau / \tau_{f}$ for three of the trajectories examined here under this assumption.

The time variation of $\Omega$ for various forcing regimes is shown in Fig. 2(c). Two peaks in $\Omega$ are observed at $\frac{t}{\tau_{f}}=\frac{1}{4}$ and $\frac{t}{\tau_{f}}=\frac{3}{4}$, predicting strongly quasisteady behavior, where the assumption that $\mathrm{St} \approx \mathrm{St}_{0}$ should hold. The characteristic frequency and Strouhal number at those two points in the cycle, which will depend on the forcing amplitude of the trajectory through $\operatorname{Re}_{q}$, can be predicted using Eq. (4) and will be investigated in the subsequent sections.

\section{Timescaling for quasisteady behavior}

It will be shown that the streamwise-oscillating cylinder displays shedding at an unsteady, timevarying, frequency, $f(t)$. Taking $\tilde{f}$ as the (constant) shedding frequency of the stationary cylinder and scaling time according to

$$
\tilde{t}=\frac{1}{\tilde{f}} \int_{0}^{t} f\left(t^{\prime}\right) d t^{\prime}
$$

gives a scaled time, $\tilde{t}$, relative to which the cylinder displays a quasisteady shedding frequency. This timescaling can be implemented by sampling data (either in experiments or postprocessing) at a rate that is constant relative to scaled time, $\tilde{t}$. Doing so in postprocessing requires temporal interpolation of the time series. Varying the sampling frequency is akin to allowing the passage of time to contract or dilate so that the observed frequency remains constant, hence the term "timescaling." Similar to $\Omega$, because the dependence of $f$ on the forcing is known then $\tilde{t}$ can be computed a priori,

$$
\tilde{t}=t+\frac{\operatorname{Re}_{q}}{\operatorname{Re}_{0} \omega_{f}}\left[\cos \left(\omega_{f} t\right)-1\right] .
$$

The scaled time, $\tilde{t}$, is shown in Fig. 2(d) for various forcing trajectories.

\section{E. Dynamic mode decomposition and timescaling}

DMD was used to extract spatiotemporally significant flow structures and gain a deeper understanding of the physical mechanisms underlying the flow. Previous authors have shown that in certain circumstances DMD modes and eigenvalues can serve as an approximation to Koopman 


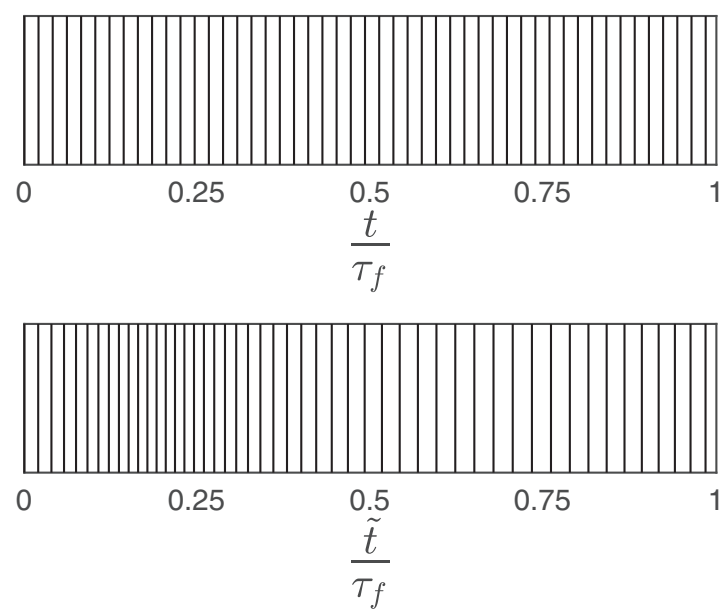

FIG. 3. Experimental sampling rate in laboratory time (top) and corresponding scaled time (bottom). Vertical lines correspond to snapshot acquisition in the experiment. For the sake of clarity, the number of samples shown has been significantly decreased relative to the actual experiment.

modes and eigenvalues [12,14,20]. This connection will be discussed further in Sec. IV C. The specific algorithm used to decompose velocity fields is detailed in Ref. [21]. DMD was first performed on PIV velocity fields acquired using a constant sampling rate relative to laboratory time, $t$, i.e., with a constant temporal separation between snapshots. Next DMD was performed on PIV velocity fields corresponding to a constant sampling rate relative to scaled time, $\tilde{t}$. The former analysis is straightforward. In the latter case, PIV snapshots do not correspond to a constant sampling rate with respect to $\tilde{t}$. The sampling rate used for PIV is shown relative to both laboratory time, $t$, and scaled time, $\tilde{t}$, in Fig. 3. However, the mapping between $t$ and $\tilde{t}$, specifically Eq. (10), is known, thus the time series were temporally interpolated to generate time series that correspond to constant sampling frequencies with respect to $\tilde{t}$. Interpolated time series will be referred to as a scaled dataset in the remainder of this work. It was observed that timescaling could lead to numerical instabilities in the corresponding dataset's DMD. Reasons for this will be elaborated on in Sec. IV but these instabilities were remedied by performing DMD using every second snapshot in the time series. Although this resulted in a lower effective sampling frequency, the effective sampling rate used for DMD was approximately 70 times higher than the stationary shedding frequency. Furthermore, DMD of unscaled datasets using all the snapshots showed little difference when compared to cases using every second snapshot for DMD. Unscaled datasets had no issues with numerical stability but DMD was still performed using every second snapshot in the time series in order to facilitate comparison with scaled data.

Phase-averaging results in a time series corresponding to a single (phase-averaged) forcing cycle. Although the temporal resolution of the time series does not change, the total length of the time series is reduced. As a result, DMD extracts a smaller number of modes and eigenvalues. DMD of phase-averaged velocity fields incorporated all the snapshots in the time series in order to increase the temporal resolution and number of resulting modes and eigenvalues. Even though double-pulse PIV velocity fields were used for phase-averaging, the effective DMD sampling rate was still approximately 25 times higher than the stationary shedding frequency.

DMD applied to a PIV time series yields both dynamic modes and eigenvalues. Eigenvalues describe the temporal behavior (i.e., growth-rate and frequency) of the spatial structure in the associated mode. In what follows, the spectral amplitudes for each dynamic mode consist of the average projection coefficient of all the snapshots in the time series onto the respective mode. As will be shown in subsequent sections, the transverse velocity field, $v$, serves as a good indicator of 


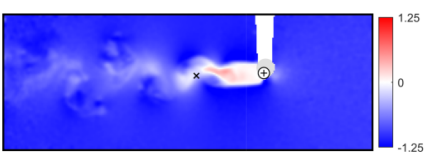

(a)

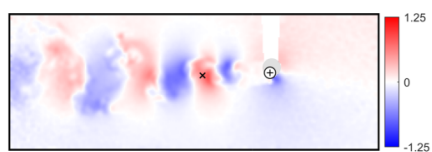

(b)

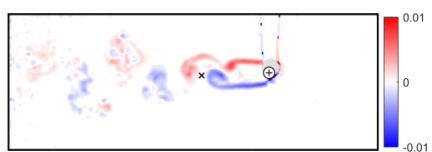

(c)

FIG. 4. Snapshots of streamwise velocity (a), transverse velocity (b), and vorticity (c) for the stationary cylinder. The cross section of the cylinder at the measurement plane is represented by $\oplus$ and the shaded circle corresponds to an obstruction in the field of view due to the bottom of the cylinder. The location of the interrogation point is denoted by $\mathbf{x}$. Flow is from right to left.

vortex shedding. Given that the shedding frequency is taken to be the characteristic frequency of the system, DMD is performed on the transverse velocity field. Vorticity is also a good indicator of shedding but computing gradients using PIV velocity fields can generate significant noise which may corrupt the DMD. Although data acquisition took place in the laboratory frame, relative to which the cylinder was moving, DMD was performed in the cylinder-fixed frame. All of the flow structures present in the wake are generated by the cylinder, hence the cylinder-fixed frame is the natural choice for analysis.

\section{RESULTS}

\section{A. Stationary cylinder}

Results for the stationary cylinder are reviewed briefly to provide the reference configuration. The instantaneous PIV velocity and vorticity fields in Fig. 4 correspond to a classic Karman vortex street. As seen in Fig. 5, the transverse component of velocity, $v$, measured at the interrogation point demonstrates an approximately constant dominant frequency, as vortices of opposite sense of rotation convect past. The power-spectral density of the transverse velocity at the interrogation point, Fig. 6(a), confirms that the shedding Strouhal number was almost identical to that predicted in the literature for this Reynolds number, $\mathrm{St} \approx 0.21$ [19]. A plot of the DMD eigenvalues for the stationary cylinder (Fig. 7) indicates that all eigenvalues lie on or inside the unit circle, such that the corresponding DMD modes either decay with time or oscillate periodically. This is true for the forced cases as well, so, for brevity, plots of eigenvalues are omitted for the rest of the cases discussed. The DMD spectrum for the stationary case is shown in Fig. 6(a), allowing a comparison of local (interrogation point) analysis and global (DMD) decomposition. Both methods have a peak near $\mathrm{St} \approx 0.2$, while the DMD spectrum for the stationary cylinder has additional, low level amplitude over a range of Strouhal numbers around the shedding peak. Comparing the DMD modes shown in Fig. 6(b) to the transverse component of velocity measured using PIV [Fig. 4(b)], the similarity between vertical structures in both the transverse velocity field and DMD modes is evident. Furthermore, the modes shown, which are identified by the colored markers on the DMD spectrum, are clustered around the shedding frequency, with the exception of the mean mode, which reflects the anticipated flow deflection around the cylinder. These DMD modes, which will henceforth be referred to as "shedding modes," are flow structures associated with vortex shedding. The finite width of the spectral peak and distribution of multiple shedding modes around it in Fig. 6(a) indicates that the shedding in the stationary case might not be purely periodic. Rather, shedding is limited to a small band of frequencies around the expected stationary value. This can likely be attributed to small fluctuations in the pump frequency driving the flow in the water channel as well as secondary instabilities present in this flow regime. It should be noted that the shedding mode [Fig. 6(b)] corresponding to the peak in the DMD spectrum, very close to the predicted shedding frequency, has the cleanest spatial shape, with minimal noise and contamination from other structures present in the flow. 

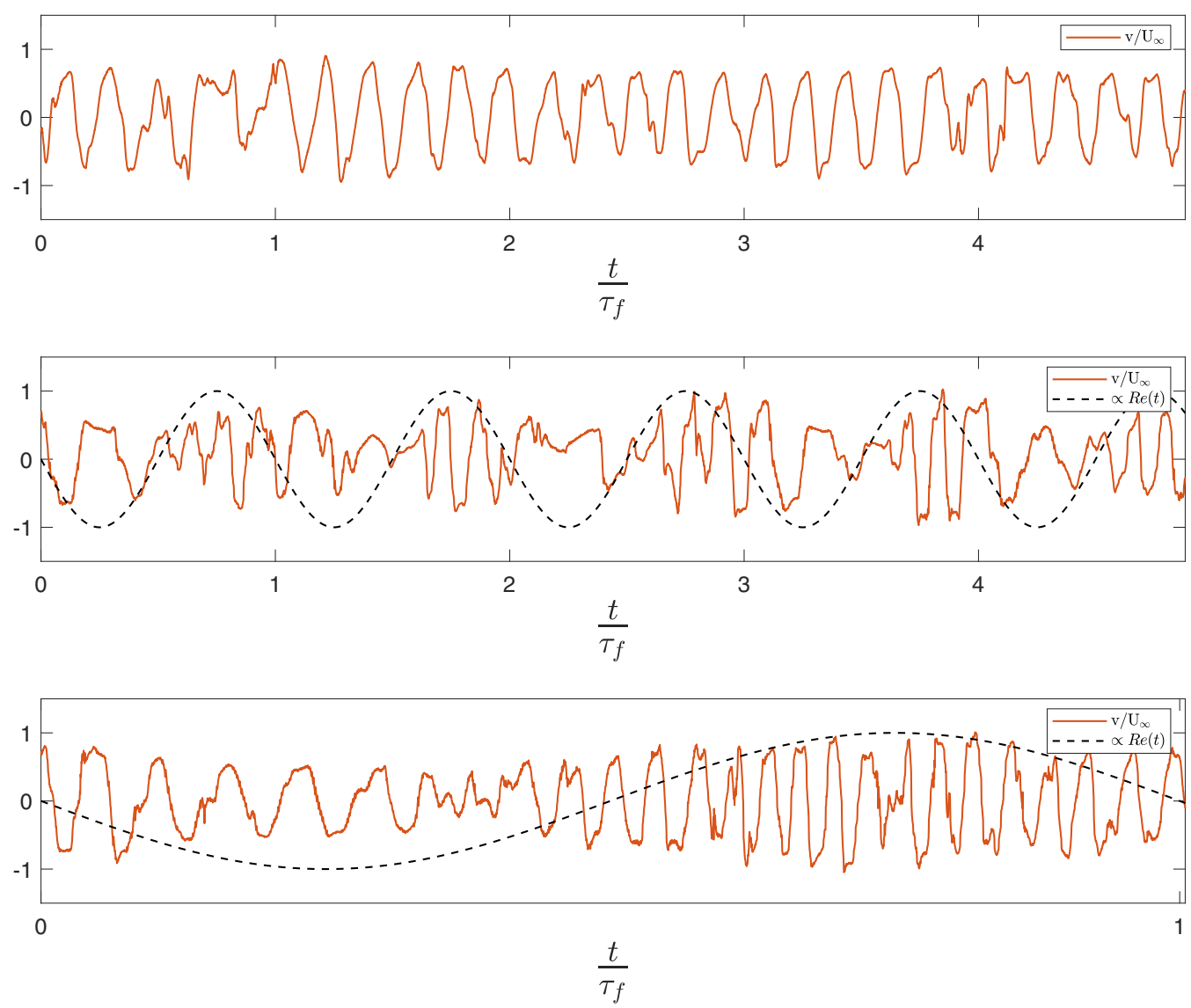

FIG. 5. Time trace of transverse velocity, $v / U_{\infty}$, at interrogation point. The dashed line is proportional to the instantaneous Reynolds number, $\operatorname{Re}(t)$. Top: Stationary cylinder; middle: $\operatorname{Re}_{0}=900, \operatorname{Re}_{q} / \operatorname{Re}_{0}=0.35$, $\mathrm{St}_{f} / \mathrm{St}_{0}=0.18$; bottom: $\operatorname{Re}_{0}=900, \mathrm{Re}_{q} / \mathrm{Re}_{0}=0.35, \mathrm{St}_{f} / \mathrm{St}_{0}=0.036$.

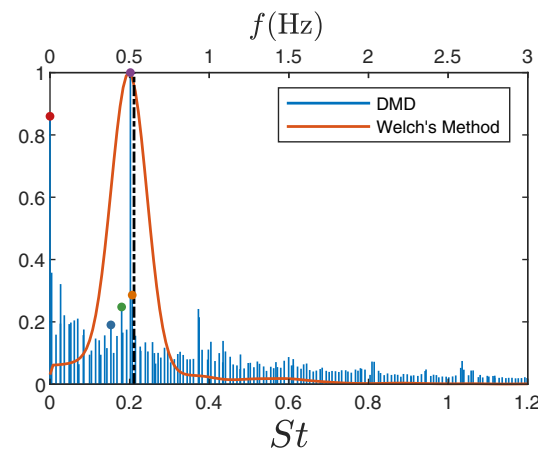

(a)

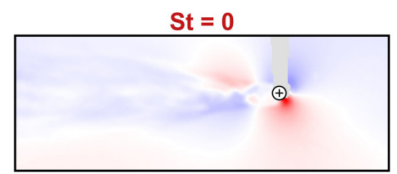

$\mathrm{St}=0.18$

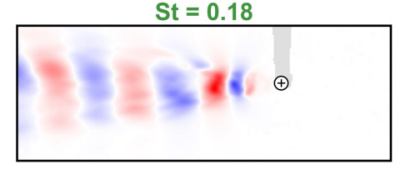

(b)

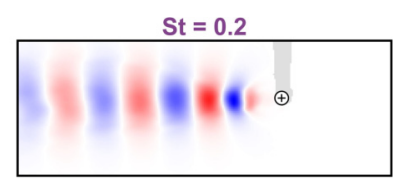

$\mathrm{St}=0.21$

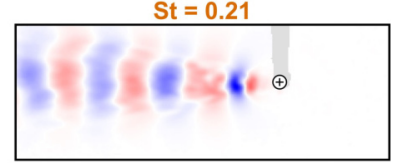

FIG. 6. DMD spectrum (a) and modes (b) for stationary cylinder, $\mathrm{Re}_{0}=900$. With the exception of the mean mode $(\mathrm{St}=0)$, the colored circles on the spectrum represent shedding modes. The colored mode labels correspond to the circles of the same color on the spectrum. The black dot-dashed line represents the stationary shedding frequency. 


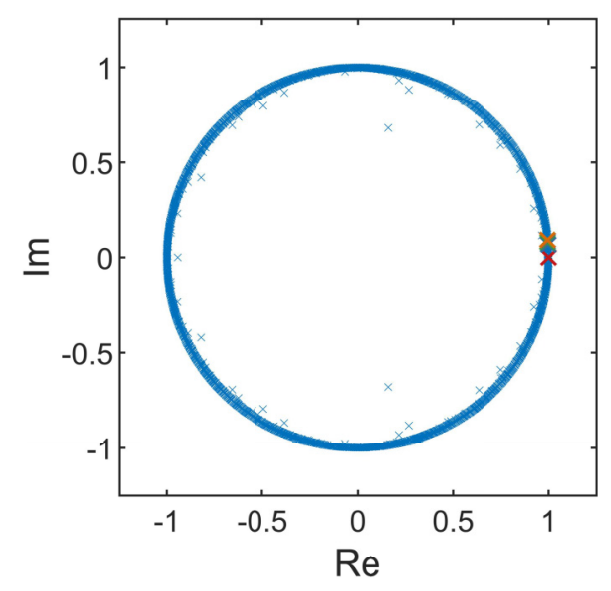

FIG. 7. DMD eigenvalues for stationary cylinder, $\operatorname{Re}_{0}=900$. The colored symbols correspond to the modes indicated in Fig. 6.

\section{B. Oscillating cylinder}

\section{Dynamic mode decomposition of the wake}

Under streamwise oscillation of the cylinder, a range of flow phenomena not present in the stationary case develop. For brevity, results are presented for two combinations of forcing parameters but similar results results were observed for the other forcing trajectories as well. Specifically, results are presented for one amplitude ratio, $\mathrm{Re}_{q} / \mathrm{Re}_{0}=0.35$, and two forcing frequency ratios, $\mathrm{St}_{f} / \mathrm{St}_{0}=0.18$ and $\mathrm{St}_{f} / \mathrm{St}_{0}=0.036$.

Snapshots of dye-flow visualization and PIV are shown for different phases of the forcing cycle in Fig. 8. The most notable effect of forcing on the wake was the spatial and temporal variation of the wake during the forcing cycle, observable as frequency and amplitude modulation of each component of velocity and out-of-plane vorticity.

The columns in Fig. 8 correspond to four different phases in the forcing cycle. From left to right, the columns correspond to $t / \tau_{f}=0,0.25,0.50$, and 0.75 , respectively. At $t / \tau_{f}=0$, the wake resembles that of the stationary cylinder with shed vortices of opposite sign convecting downstream. Although the larger-scale structure of the wake is similar to the stationary case, streamwise forcing also generates smaller-scale structures and unsteadiness which can be seen in the velocity and vorticity fields. The cylinder reaches maximum downstream velocity when $t / \tau_{f}=0.25$. It can be seen that the strength of shed vortices and the corresponding shear layers have decreased at this point in the forcing cycle. Although the instantaneous freestream velocity seen by the cylinder at $t / \tau_{f}=0$ and 0.50 is identical, the wake at the upstream turnaround point is not identical to its downstream counterpart. In the latter case, the wake no longer resembles that of the stationary cylinder. At $t / \tau_{f}=0.50$, the wake is relatively quiescent and the near wake sees the development of two symmetric shear layers which roll up into a pair of symmetric vortices. The behavior at the downstream turnaround point will be discussed in more detail in Sec. III B 2. At $t / \tau_{f}=0.75$, the cylinder attains its maximum upstream velocity and also reaches its most active state. At this point in the forcing trajectory vortex shedding has resumed and the increase in instantaneous freestream velocity seen by the cylinder leads to stronger shear layers and shed vortices. The modulation in strength of shed vortices is also observed at the interrogation point in the form of the amplitude modulation, as seen in Fig. 5.

More detail can be observed in the time traces at the interrogation point, shown in Fig. 5. The amplitude modulation was stronger for cases corresponding to $\mathrm{St}_{f}<\mathrm{St}_{0}$ while the frequency modulation was more pronounced for cases where $\mathrm{St}_{f} \ll \mathrm{St}_{0}$. It is important to note, however, that 

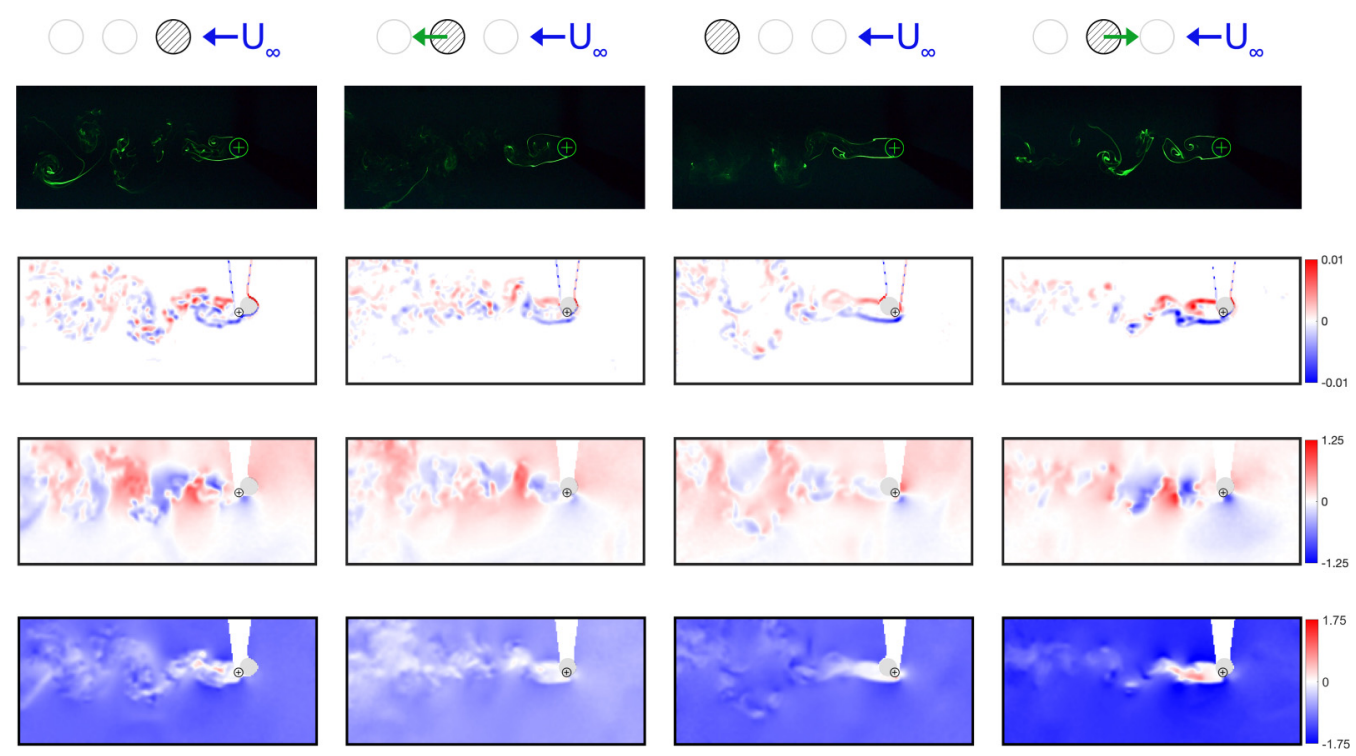

FIG. 8. Flow around a streamwise oscillating cylinder, $\mathrm{Re}_{0}=900, \mathrm{Re}_{q} / \mathrm{Re}_{0}=0.35, \mathrm{St}_{f} / \mathrm{St}_{0}=0.18$. The top row corresponds to the position of the cylinder in the forcing cycle. The second row corresponds to visualization of the flow using fluorescent dye. The third row corresponds to vorticity $\omega_{z}$. The fourth and fifth rows correspond to the transverse and streamwise components of velocity, $\frac{v}{U_{\infty}}$ and $\frac{u}{U_{\infty}}$, respectively. From left to right, the columns correspond to $t / \tau_{f}=0,0.25,0.50$, and 0.75 , respectively. The $\bigoplus$ symbol represents the cross section of the cylinder at the measurement plane while the shaded circle represents an obstruction in the field of view due to the bottom of the cylinder. Flow is from right to left.

both forcing frequencies still lead to amplitude and frequency modulation, to varying extents and correlated with the instantaneous Reynolds number seen by the cylinder, shown schematically by the dashed line as a reference to when various flow phenomena occur relative to the phase of the forcing cycle. It can be seen that shedding at points corresponding to $t \approx \frac{1}{4} \tau_{f}+k \tau_{f}$ and $t \approx \frac{3}{4} \tau_{f}+k \tau_{f}$, where $k=0,1,2,3, \ldots$, was the most consistent. That is, shedding occurred at a roughly constant frequency at those points in the forcing cycle. Those points in the cycle also correspond to peaks in $\Omega$ where strong quasisteady behavior is expected, see Sec. II B.

The PSDs for both forcing regimes [Figs. 9(a) and 10(a)] are broader than the stationary case, reflecting the enhanced vortical activity in the wake, with the development of two distinct peaks especially clear for the lower forcing frequency. Changes are also observed in the DMD spectra. When $\operatorname{Re}_{q}$ is fixed but forcing frequency is decreased by an order of magnitude, additional shedding modes are extracted from DMD of the PIV time series. These additional shedding modes arise because of the gradual modulation of shedding frequency during a forcing period. Four representative DMD modes are shown in Figs. 9(b) and 10(b) for each case, selected to reflect the mean, a mode close to the forcing frequency, and the strongest shedding modes in each spectral peak. In each case, the latter two panels show similar features to the stationary cylinder shedding mode, but at a modified frequency. A similar phenomena was presented by Arbabi and Mezic [22] who observed the persistence of structurally similar Koopman modes in lid driven cavity flow over a wide range of Reynolds numbers. The zero frequency "mean modes" also resemble their stationary counterpart in that they contain an antisymmetric structure in the near wake. Relative to the stationary case, however, streamwise forcing generates small variations in strength and structure of the mean modes.

It was also observed that the peak in the DMD spectrum widened with forcing amplitude (not shown), a phenomenon also seen by Glaz et al. [7]. Considering cases with different forcing amplitudes, $\operatorname{Re}_{q}$, but all other parameters held constant, it is observed that the separation between 


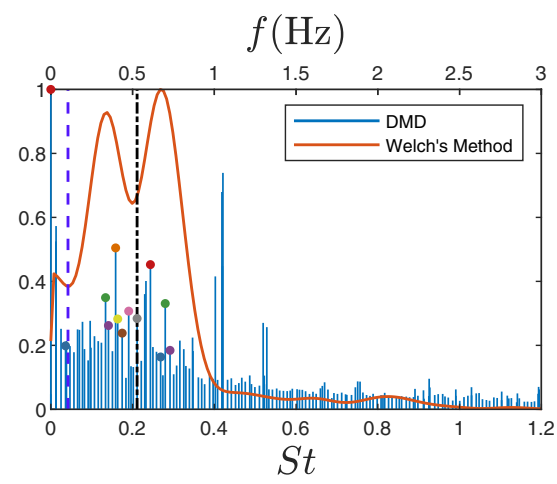

(a)

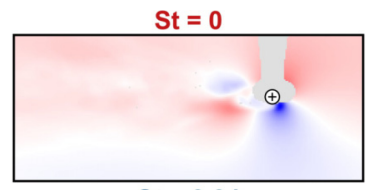

$\mathrm{St}=0.04$

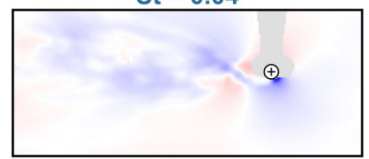

(b)

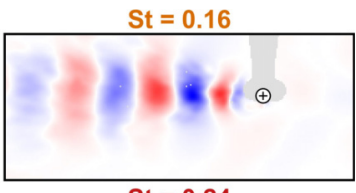

$\mathrm{St}=0.24$

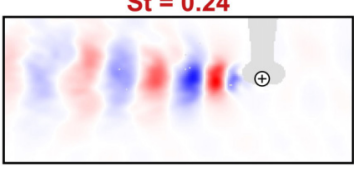

FIG. 9. DMD spectrum (a) and modes (b) for oscillating cylinder, $\operatorname{Re}_{0}=900, \mathrm{Re}_{q} / \mathrm{Re}_{0}=0.35, \mathrm{St}_{f} / \mathrm{St}_{0}=$ 0.18. The colored mode labels correspond to the circles of the same color on the spectrum. The black dotdashed line represents the stationary shedding frequency while the purple dashed line denotes the forcing frequency. The additional colored symbols on the spectrum indicate shedding modes.

spectral peaks increases with forcing amplitude for both the interrogation point and DMD spectra. The development of a relatively strong but narrow outlying peak near $\mathrm{St} \approx 0.4$ in the DMD spectra shown in Fig. 9(a) does not play a significant role in the system's dynamics. The outlying peak corresponds to a transient set of mode pairs with stable eigenvalues indicating decaying mode contribution with increasing time. The mode pairs also contain similar spatial structures (displaying weak coherence and significant noise) but are almost perfectly out of phase resulting in a very small net contribution from the mode pairs to the overall system dynamics. The peak near $\mathrm{St} \approx 0.5$ is ignored for the same reason.

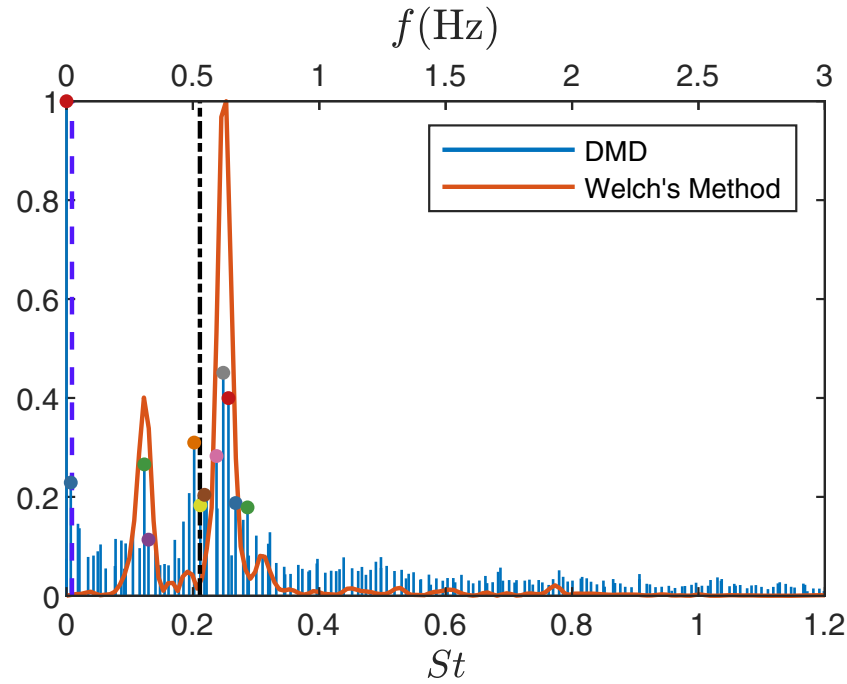

(a)

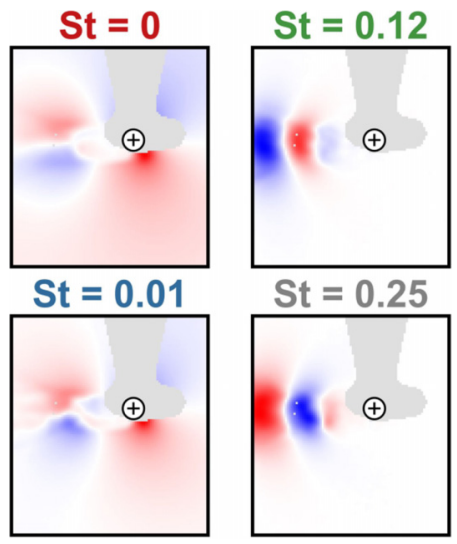

(b)

FIG. 10. DMD spectrum (a) and modes (b) for oscillating cylinder, $\mathrm{Re}_{0}=900, \mathrm{Re}_{q} / \mathrm{Re}_{0}=0.35, \mathrm{St}_{f} / \mathrm{st}_{0}=$ 0.036 . 


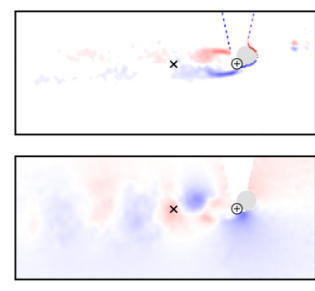

(a)

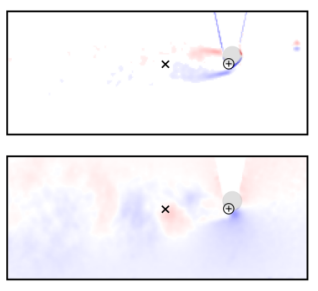

(b)

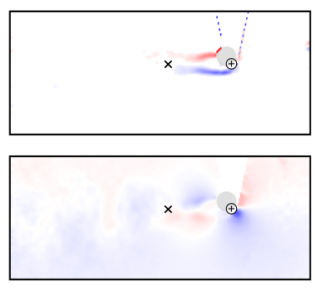

(c)

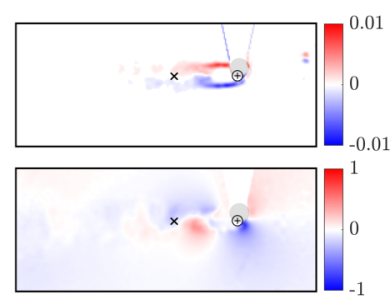

(d)

FIG. 11. Phase-averaged vorticity (top) and transverse velocity (bottom), $\operatorname{Re}_{0}=900, \operatorname{Re}_{q} / \mathrm{Re}_{0}=0.35$, $\mathrm{St}_{f} / \mathrm{St}_{0}=0.18$. (a) $t / \tau_{f}=0$; (b) $t / \tau_{f}=0.25$; (c) $t / \tau_{f}=0.50$; (d) $t / \tau_{f}=0.75$.

\section{Structure generated at the forcing frequency}

Besides the wake structure discussed above, the forcing frequency itself imposes temporal structure on the flow field. This can be recovered by phase-averaging the flow fields, shown here at a series of phases during the forcing cycle for $\mathrm{Re}_{q} / \mathrm{Re}_{0}=0.35$ and $\mathrm{St}_{f} / \mathrm{St}_{0}=0.18$.

Vorticity fields corresponding to the phase-averaged time series are presented in Fig. 11 for different phases in the cycle. The four snapshots of phase-averaged vorticity show the effect of forcing on shear-layer strength and spacing. As the cylinder moves downstream and the instantaneous freestream velocity seen by the cylinder decreases, the shear layers weaken and move apart from each other. On the other hand, as the cylinder moves upstream, the instantaneous freestream velocity seen by the cylinder increases, and the shear layers strengthen. This leads to the contraction and narrowing of the wake. As expected, phase-averaging reduces the observability of vortex shedding in the wake, the period for which is neither constant nor related to the forcing period through an integer number of cycles.

The streamlines of the phase-averaged flow, shown in Fig. 12, reveal a striking feature as the cylinder approaches the downstream turnaround point and changes direction, corresponding to $t=$ $\frac{1}{2} \tau_{f}+k \tau_{f}$. Near the downstream turnaround point, the shear layers on either side of the cylinder become straight as the local Reynolds number reaches a minimum and shedding ceases. Initially smooth streamlines close in a symmetric manner about the wake centerline, and then a symmetric structure develops as the forcing cycle advances and the cylinder starts to move upstream, before asymmetric shedding resumes. The development of closed streamlines alludes to the generation of two symmetric vortices downstream of the body with one on either side of the cylinder centerline,

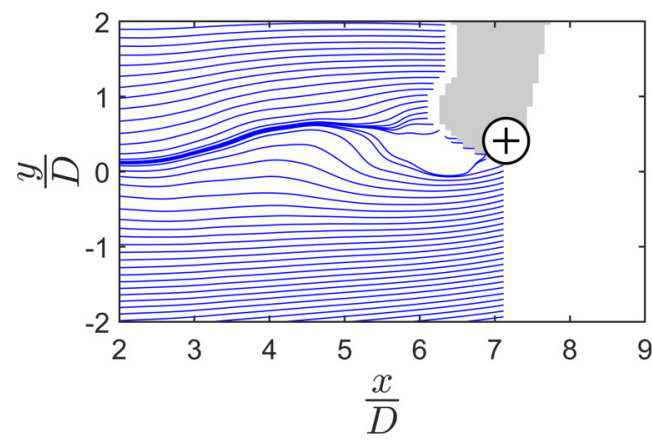

(a)

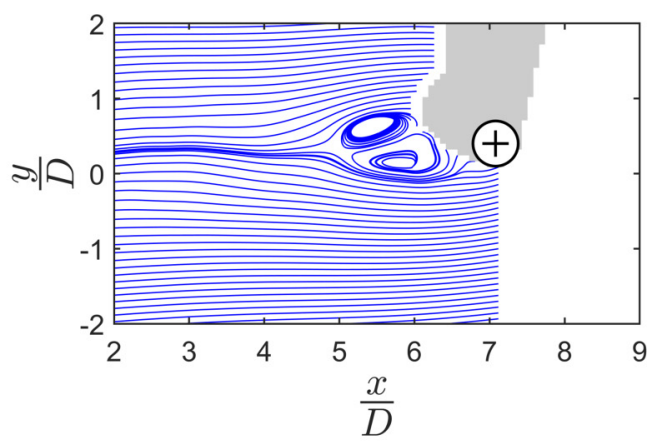

(b)

FIG. 12. Streamlines for phase-averaged velocity field: Before formation of starting vortex, $\frac{t}{\tau_{f}}=0.4$ (a); during formation of starting vortex, $\frac{t}{\tau_{f}}=0.5$ (b). $\mathrm{Re}_{0}=900, \mathrm{Re}_{q} / \mathrm{Re}_{0}=0.35, \mathrm{St}_{f} / \mathrm{St}_{0}=0.18$. 


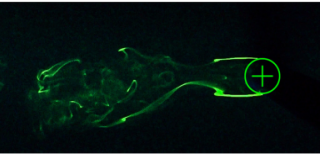

(a)

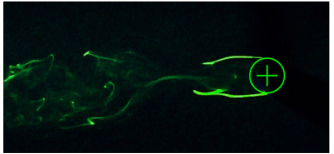

(b)

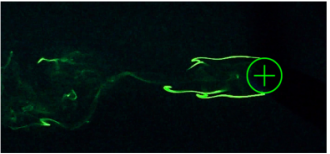

(c)

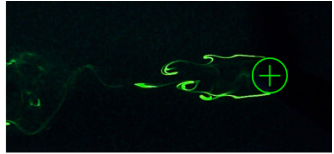

(d)

FIG. 13. Dye flow visualization of starting vortex generation. (a) $t / \tau_{f}=0.50$; (b) $t / \tau_{f}=0.55$; (c) $t / \tau_{f}=$ $0.60 ;(\mathrm{d}) t / \tau_{f}=0.65$.

as seen clearly in the (instantaneous) dye-flow visualization of Fig. 13. While the phase-averaged closed streamlines at $t / \tau_{f}=0.50$ in Fig. 12 also resemble the mean recirculation bubble in the wake of a stationary cylinder, the generation of this symmetric pattern (present for only a short duration) and the related roll up of dye filaments are anchored to the same phase in the forcing cycle and we refer to this as the development of "starting vortices," highlighting the near wake's resemblance to that of an impulsively started cylinder.

The dynamic mode decomposition of the phase-averaged velocity fields is presented in Fig. 14 (recall that the phase-averaging implemented here leads to a lower frequency resolution in the DMD spectrum). Phase-averaging leads to a reduction in activity in the frequency range for vortex shedding, a trend which should continue with increasing record length. The emergence of an antisymmetric flow structure at the forcing frequency can be identified from Fig. 14(b). This DMD mode will be referred to as the "forcing mode." Footprints of the antisymmetric forcing mode are also seen in the DMD presented above which were not phase-averaged [Fig. 9(b) and Fig. 10(b)]. It has previously been shown that the phase-averaged velocity field is directly related to the Koopman mode at the averaging frequency [23]. Hence, the forcing mode extracted in the phase-averaged case is possibly related to the dynamics of the non-phase-averaged system via this connection with the Koopman operator.

\section{Quasisteady scaling}

The flow field and DMD are now considered under the quasisteady timescaling introduced in Sec. III. Figure 15 shows the time traces of Fig. 5, which exhibited strong frequency modulation, under timescaling, i.e., with respect to $\tilde{t}$. For both forcing regimes an approximately constant shedding frequency can be observed, which is particularly evident for $\mathrm{St}_{f} \ll \mathrm{St}_{0}$. This observation is confirmed by the PSDs in Fig. 16 and Fig. 17, which show a single peak and significant narrowing from the two peak spectra of Fig. 9(a) and Fig. 10(a), respectively.

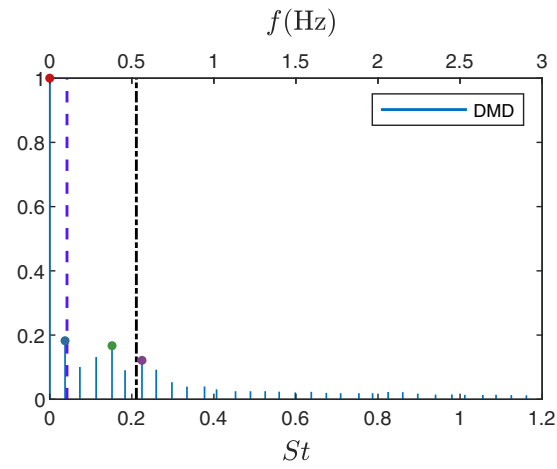

(a)

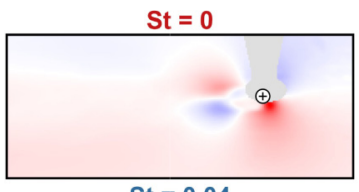

St $=0.04$

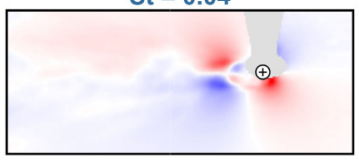

(b)

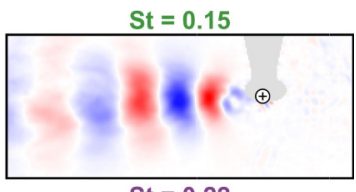

St $=0.22$

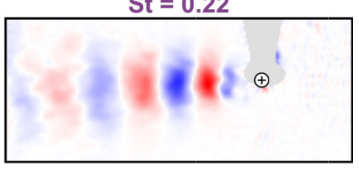

FIG. 14. DMD spectrum (a) and modes (b) for phase-averaged time series, $\operatorname{Re}_{0}=900, \operatorname{Re}_{q} / \mathrm{Re}_{0}=0.35$, $\mathrm{St}_{f} / \mathrm{St}_{0}=0.18$. 

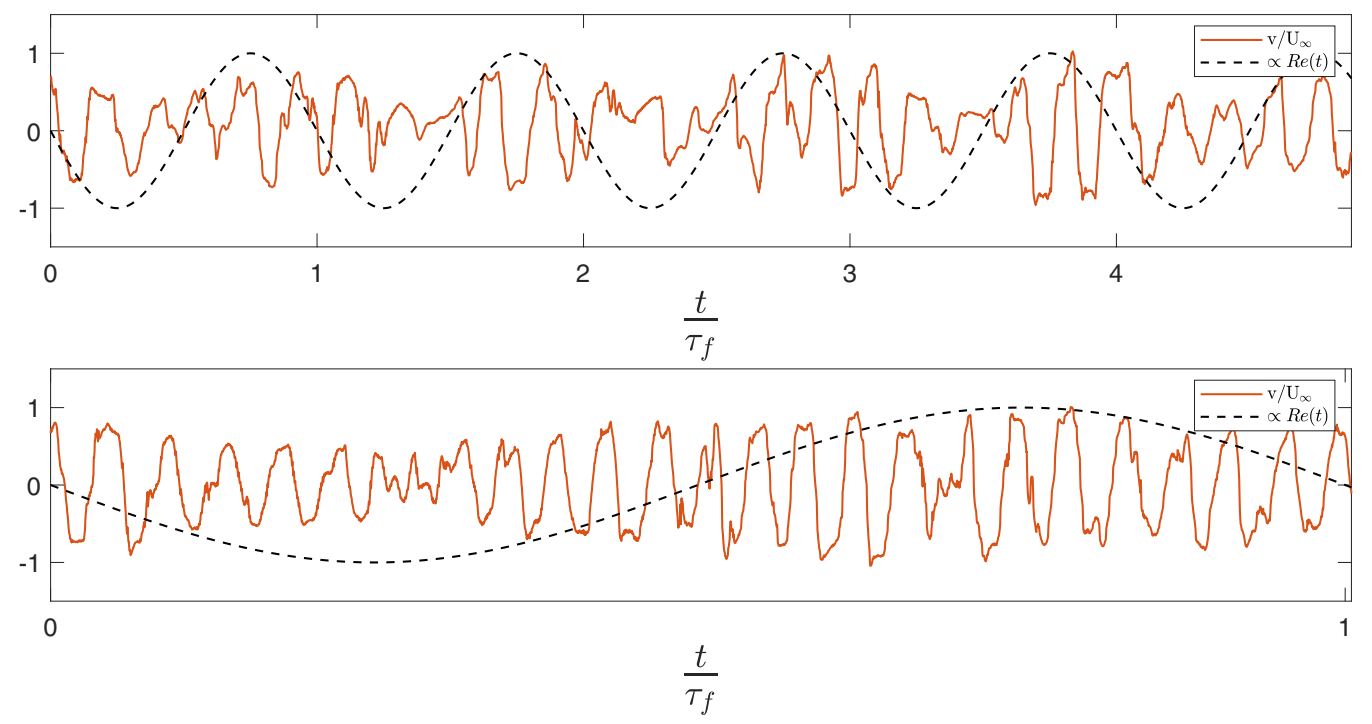

FIG. 15. Time trace of transverse velocity, $v / U_{\infty}$, at interrogation point with quasisteady timescaling. Top: $\mathrm{Re}_{0}=900, \mathrm{Re}_{q} / \mathrm{Re}_{0}=0.35, \mathrm{St}_{f} / \mathrm{st}_{0}=0.18 ;$ bottom: $\mathrm{Re}_{0}=900, \mathrm{Re}_{q} / \mathrm{Re}_{0}=0.35, \mathrm{St}_{f} / \mathrm{st}_{0}=0.036$.

DMD of the scaled data, presented in Figs. 16 and 17, also shows that the two peaks present in the DMD spectra of the unscaled cases are no longer present. Furthermore, DMD extracts fewer shedding modes which are located in a smaller band of frequencies when compared to the unscaled DMD. In addition to the collapse of shedding modes onto a single peak, the prominence of the peak is also increased relative to the unscaled case. While the quasisteady scaling seems to be relatively successful in identifying a simpler structure to the vortex shedding, it cannot treat the development of the starting vortices.

\section{DISCUSSION}

The previous section has identified the development of numerous unsteady phenomena in the streamwise-oscillating cylinder wake, including frequency modulation, amplitude modulation, and

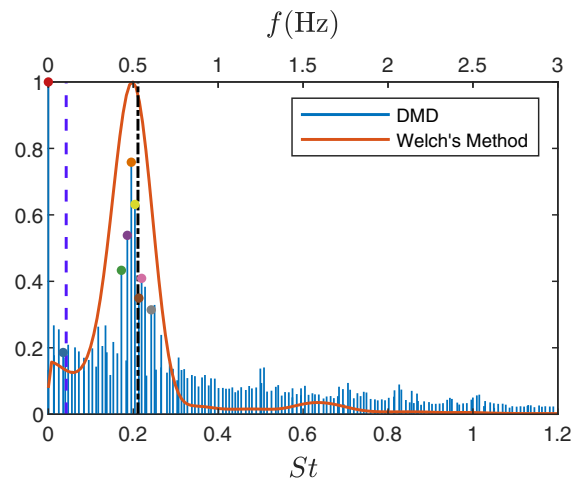

(a)

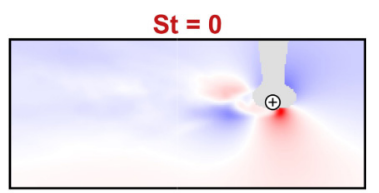

St $=0.03$

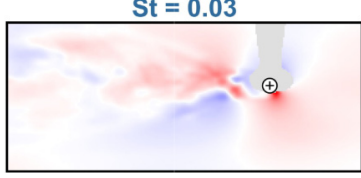

(b)

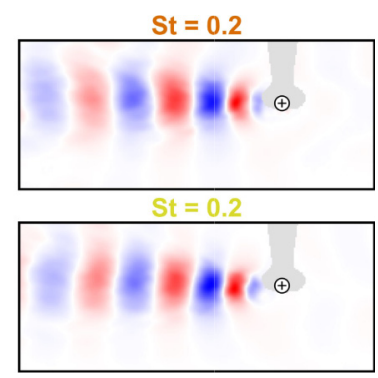

FIG. 16. DMD spectrum (a) and modes (b) for oscillating cylinder with quasisteady timescaling, $\operatorname{Re}_{0}=$ $900, \mathrm{Re}_{q} / \mathrm{Re}_{0}=0.35, \mathrm{St}_{f} / \mathrm{St}_{0}=0.18$. 


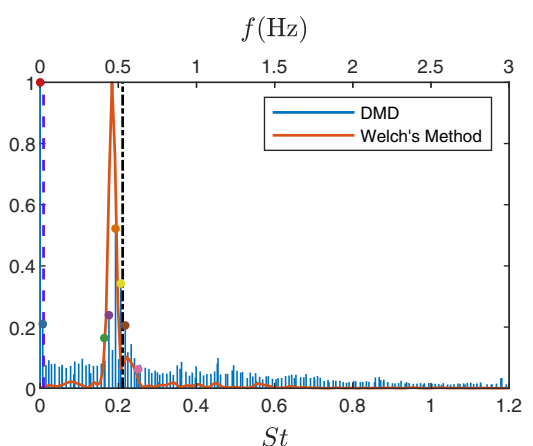

(a)

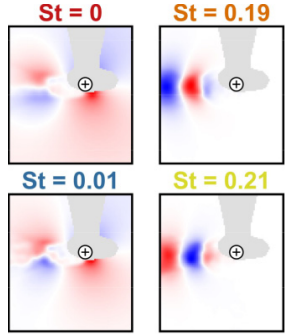

(b)

FIG. 17. DMD spectrum (a) and modes (b) for oscillating cylinder with quasisteady timescaling, $\mathrm{Re}_{0}=$ $900, \mathrm{Re}_{q} / \mathrm{Re}_{0}=0.35, \mathrm{St}_{f} / \mathrm{st}_{0}=0.036$.

the generation of starting vortices. The complexity of this flow can be reduced by quasisteady timescaling, the success of which hinges on two factors. First, the characteristic (shedding) frequency of the stationary cylinder must be primarily governed by the Reynolds number. Second, the separation of scales between forcing and shedding frequency facilitates the development of quasisteady shedding during certain portions of the forcing cycle. These two factors connect the forcing trajectory to the frequency modulation observed.

\section{A. Quasisteady shedding regimes}

As presented in Sec. II C, the system is predicted to show strong quasisteady behavior when $\Omega$ is large. Using Eq. (4) the shedding frequency corresponding to each of the two peaks in $\Omega$ can be predicted. In Fig. 18 the observed spectral peaks for the interrogation point are compared with the (predicted) dominant Strouhal number corresponding to each peak in $\Omega$. The peaks in the interrogation point spectra are in good agreement with the prediction indicating that the two peak nature of the unscaled PSDs can be identified with periods of large $\Omega$ and that the observed dominant frequencies arise due to quasisteady behavior.

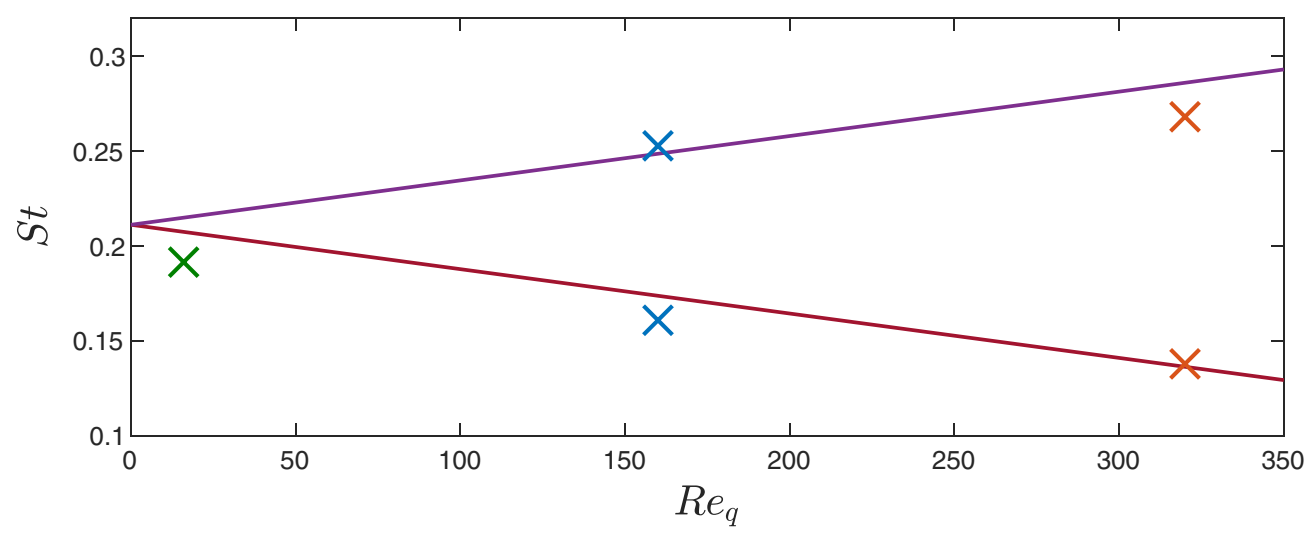

FIG. 18. Comparison of predicted and observed dominant frequencies, $\operatorname{Re}_{0}=900, \mathrm{St}_{f} / \mathrm{St}_{0}=0.18$. The solid lines represent the predicted dominant frequencies while the $\times$ symbols represent the actual dominant frequencies observed in experiments. 

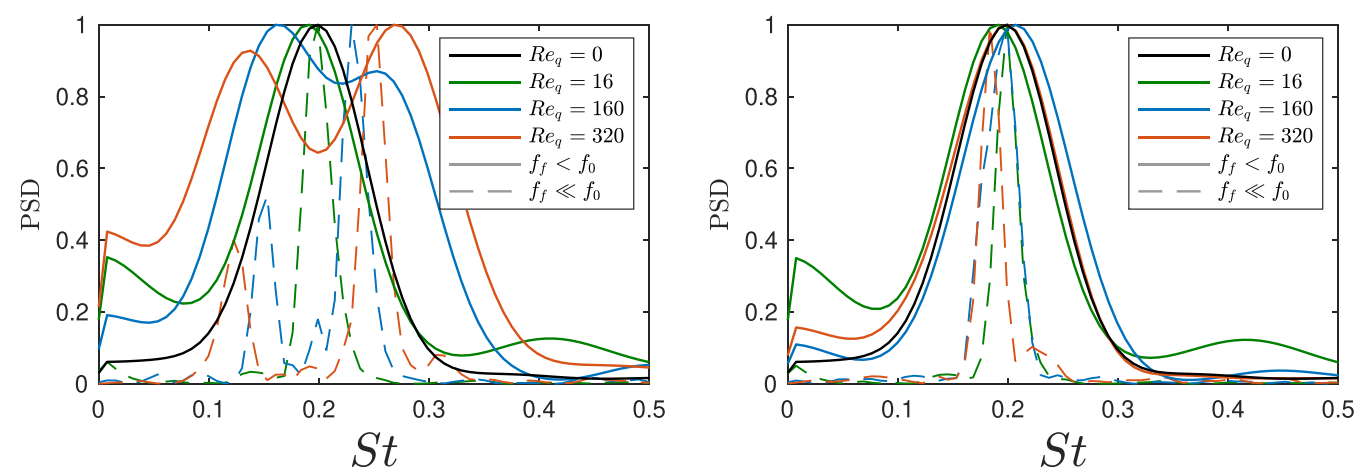

FIG. 19. Comparison of interrogation point power spectra before (left) and after (right) application of quasisteady timescaling.

Although the dominant frequencies predicted in Fig. 18 are in good agreement with observed spectra, the assumptions were rather crude, thus a few factors may be underlying any discrepancies. First, the spectra were calculated using Welch's method, which depends heavily on window quantity and size. Varying these parameters can lead to different estimates of PSD. Thus, these parameters may cause the interrogation point spectra to deviate from the prediction. Although $\mathrm{St}_{0}$ is approximately constant for the range of Reynolds numbers encountered, it can vary by up to $3 \%$ during the forcing cycle, a deviation which may percolate through the analysis. It should also be noted timescaling is applied uniformly to each snapshot in the time series. That is, the scaling procedure does not account for the fact that it takes a finite amount of time for vortical structures to convect downstream. Even still, the quasisteady timescaling gives reasonable collapse of the two peaks in the measured spectra onto a single spectral peak. Finally, it should be mentioned that the scaling procedure does not account for the vortex interactions, shear-layer dynamics, and resulting amplitude modulation discussed above. These factors may further cause deviations from the prediction. For example, the modulation of shear-layer strength is such that starting vortices may develop at times close to peaks in $\Omega$, where shedding at the dominant frequencies is expected.

Beyond the presence of two peaks in $\Omega$ during the forcing cycle, Fig. 2(c) also displays an asymmetry between the two. $\dot{f}$ governs the location of the peaks and is controlled by the cylinder acceleration; however, $\dot{\tau}$ [Eq. (5)] also includes the instantaneous shedding frequency, which depends on the sense of the cylinder motion relative to the freestream, giving rise to the asymmetry. This asymmetry is an important distinction because the wider peak corresponds to the most active portion of the forcing trajectory where shedding is stronger. In this context, "stronger" refers to the duration of the quasisteady portion, strength of shed vortices, and relative magnitude of each spectral peak.

Due to the continuous change in shedding frequency along a forcing trajectory, DMD of the unscaled system extracts additional shedding modes at multiple frequencies, in addition to those at the two dominant frequencies. This means that the dynamics of the unscaled system are not restricted to a small number of shedding modes and frequencies, even though the shape and structure of the shedding modes are quite similar. After applying the timescaling, spectral peaks from a range of forcing amplitudes, $\operatorname{Re}_{q}$, collapse onto each other exhibiting quasisteady behavior, as shown in Fig. 19. The peaks are narrower for $\mathrm{St}_{f} \ll \mathrm{St}_{0}$.

\section{B. Amplitude modulation and partial lock-on}

Although the forcing trajectory leads to some amplitude modulation in the wake, the mechanism governing this behavior is not identical to that responsible for frequency modulation. As presented above, the development of symmetric starting vortices and amplitude modulation are both phase- 
locked to the forcing. Furthermore, phase-averaged vorticity fields show that the modulation of shear-layer strength is also phase-locked to the forcing. Consideration of the relative freestream velocity seen by the cylinder helps elucidate the cause of this phenomena. As the cylinder moves downstream the relative freestream velocity seen by the cylinder decreases and so does the strength of the shear layer. The cylinder follows a sinusoidal forcing trajectory so as it starts to approach the downstream turnaround point, it slows down, causing an increase in the relative freestream velocity seen by the cylinder. Consequently the shear-layer strength begins to increase. When the shear layer becomes sufficiently strong, starting vortices are generated. As the starting vortices develop, asymmetric shedding ceases, and the wake is relatively quiescent (i.e., oscillatory behavior and transverse velocity are suppressed). Thus, the spatiotemporal variation of the shedding type and strength leads to amplitude modulation in the wake.

The range of forcing frequencies considered in this study do not overlap with those required for the canonical lock-on phenomena. The generation of starting vortices at the downstream turnaround point, however, is synchronized with the forcing. In contrast to the classical definition of lock-on in which the vortex shedding frequency is proportional to the forcing frequency, the generation of starting vortices can be thought of as a "pseudo" or "partial" lock-on phenomenon. Specifically, the generation frequency of starting vortices is proportional to the forcing frequency even though the vortex shedding frequency is not.

\section{Connections with the Koopman operator}

An important nuance in using DMD arises from its assumption of linear dynamics. Namely, DMD gives the eigendecomposition of the of the best-fit linear operator that approximates system dynamics. For a system governed by linear dynamics, the connection between the decomposition and observed behavior is clear. The utility of applying this linear technique to a nonlinear system, however, is not obvious. Namely, the nonlinearity of the Navier-Stokes equations adds a level of ambiguity when relating DMD to the underlying flow physics. The interpretation of DMD as an approximation of the Koopman modes and eigenvalues has often been used to bolster this relation. As outlined above, the Koopman operator is a linear, infinite-dimensional composition operator enabling the analysis of nonlinear systems from a linear perspective. Although DMD has strong connections to the Koopman operator, resulting modes and eigenvalues do not always represent their Koopman counterparts and, in particular, do not always allow for the identification of the full set of Koopman eigenmodes. Although variations of DMD, such as Extended DMD [20], have been developed to exploit this connection, Drmac et al. [14] present another point of tangency. They show that under certain conditions, DMD does indeed suffice as a good approximation of the Koopman operator's eigendecomposition. In the context of the current study, if the wake dynamics settle on a finite-dimensional nonlinear attractor embedded in an infinite-dimensional space, then linear observables, such as velocity, suffice and DMD provides a reasonable approximation of the Koopman mode decomposition [14]. The periodicity of observed wake structures and forcing alludes to such a state. Thus, the DMD modes and eigenvalues are expected to approximate the respective Koopman mode decomposition. This further strengthens the validity of using DMD to study the streamwise-oscillating cylinder as it solidifies the link between the observed nonlinear dynamics and extracted flow structures. This connection can be verified further using extended DMD but is left for future work.

\section{CONCLUSION}

The flow around streamwise-oscillating cylinders at moderate Reynolds number was studied. Forcing frequencies one and two orders of magnitude less than the stationary shedding frequency were considered, well below the frequency ratios corresponding to lock-on. Various amplitude ratios were also studied, all satisfying the requirement that the instantaneous Reynolds number remain above the critical value. Experiments were carried out in a closed loop water tunnel in order to 
assess the effect of streamwise forcing on the wake. Particle image velocimetry was used to obtain snapshots of velocity while fluorescent dye was used to illuminate flow structures that developed due to streamwise forcing. Experiments showed that streamwise forcing led to the development of both frequency and amplitude modulation in the wake. Additionally, a range of vortex interactions were observed including the development of symmetric starting vortices.

Dynamic mode decomposition was used to extract spatiotemporally significant flow structures from experimental velocity fields. This revealed that vortex shedding lead to the generation of shedding modes at the corresponding shedding frequency. Furthermore, it was seen that as the amplitude of oscillation increased, the number of shedding modes extracted using DMD followed suit. Global spectra from DMD revealed a spread in the spectral distribution of shedding modes. Additionally, interrogation point spectra revealed the development of two spectral peaks, indicating the presence of two dominant frequencies in the streamwise-oscillating cylinder's wake.

Phase-averaging was used to show that shear-layer strength was phase-locked to the forcing. It was shown that the modulation of shear-layer strength lead to the development of symmetric starting vortices at the downstream turnaround point. Consequently, oscillatory behavior in the wake was suppressed, leading to amplitude modulation. DMD of the phase-averaged flow fields resulted in the extraction of a forcing mode corresponding to the modulation of shear-layer strength.

In addition to experiments, analysis was used to show that the forcing frequency allowed for the development of quasisteady dynamics at various points in the forcing trajectory. A parameter, the quasisteadiness, was developed in order to predict when quasisteady dynamics would be present and was in good agreement with experiments. Furthermore, it was shown that the shedding frequency of the system could be predicted for portions of the forcing cycle exhibiting quasisteady behavior. Using the interplay between unsteady and quasisteady dynamics along the forcing trajectory, a transformation was developed to scale time and normalize the characteristic frequency of the system. That is, timescaling was used to transform the system's dynamics from unsteady to quasisteady. Timescaling applied to experimental velocity fields showed that both the DMD and interrogation point spectra collapsed onto a single spectral peak. Furthermore, fewer shedding modes were extracted, indicating that vortex shedding was occurring in smaller band of frequencies. Thus, in contrast to the unscaled case, timescaling produced a more meaningful dynamic mode decomposition that could be clearly be related back to system dynamics.

It was shown that constant sampling relative to the laboratory time, $t$, resulted in an irregular sampling rate relative to the scaled time, $\tilde{t}$. Going forward, experiments can be performed with constant sampling relative to the scaled time in order to bypass the need for timescaling as a step in postprocessing. It is worth noting that the analysis presented in this work is not limited to fluid systems, but rather it is applicable to any periodic system with a characteristic frequency determined by a set of governing parameters. Many systems of engineering and scientific interest display periodic behavior. Of these, multiple have well established connections between governing parameters and frequency. These systems may indeed be amenable to the timescaling and analysis presented here.

\section{ACKNOWLEDGMENTS}

This work was supported by U.S. Army Research Office Grant No. W911NF-17-1-0306. The authors also thank Alexandra Techet, Shai Revzen, and Morgan Hooper for insightful discussions and input.

[1] C. Williamson and A. Roshko, Vortex formation in the wake of an oscillating cylinder, J. Fluids Struct. 2, 355 (1988).

[2] C. Williamson and R. Govardhan, Vortex-induced vibrations, Annu. Rev. Fluid Mech. 36, 413 (2004). 
[3] C. Barbi, D. Favier, C. Maresca, and D. Telionis, Vortex shedding and lock-on of a circular cylinder in oscillatory flow, J. Fluid Mech. 170, 527 (1986).

[4] J. Leontini, D. Jacono, and M. Thompson, A numerical study of an inline oscillating cylinder in a freestream, J. Fluid Mech. 688, 551 (2011).

[5] J. Leontini, D. Jacono, and M. Thompson, Wake states and frequency selection of a streamwise oscillating cylinder, J. Fluid Mech. 730, 162 (2013).

[6] E. Konstantinidis, D. Dorogi, and L. Baranyi, Resonance in vortex-induced in-line vibration at low reynolds numbers, J. Fluid Mech. 907, A34 (2020).

[7] B. Glaz, I. Mezić, M. Fonoberova, and S. Loire, Quasi-periodic intermittency in oscillating cylinder flow, J. Fluid Mech. 828, 680 (2017).

[8] O. Griffin and S. Ramberg, Vortex shedding from a cylinder vibrating in line with an incident uniform flow, J. Fluid Mech. 75, 257 (1976).

[9] C. Rowley and S. Dawson, Model reduction for flow analysis and control, Annu. Rev. Fluid Mech. 49, 387 (2017).

[10] K. Taira, S. Brunton, S. Dawson, C. Rowley, T. Colonius, B. McKeon, O. Schmidt, S. Gordeyev, V. Theofilis, and L. Ukeiley, Modal analysis of fluid flows: An overview, AIAA J. 55, 4013 (2017).

[11] P. J. Schmid, Dynamic mode decomposition of numerical and experimental data, J. Fluid Mech. 656, 5 (2010).

[12] C. W. Rowley, I. Mezić, S. Bagheri, P. Schlatter, and D. S. Henningson, Spectral analysis of nonlinear flows, J. Fluid Mech. 641, 115 (2009).

[13] K. K. Chen, J. H. Tu, and C. W. Rowely, Variants of dynamic mode decomposition: Boundary condition, koopman, and fourier analyses, J. Nonlin. Sci. 22, 887 (2012).

[14] Z. Drmač, I. Mezić, and R. Mohr, Data driven modal decompositions: Analysis and enhancements, SIAM J. Sci. Comput. 40, A2253 (2018).

[15] L. A. Segel and M. Slemrod, The quasi-steady-state assumption: A case study in perturbation, SIAM Rev. 31, 446 (1989).

[16] T. van Cutsem and C. Vournas, Voltage Stability of Electric Power Systems (Springer US, New York, 1998), pp. 166-167.

[17] S. P. Sane and M. H. Dickinson, The aerodynamic effects of wing rotation and a revised quasi-steady model of flapping flight, J. Exp. Biol. 205, 1087 (2002).

[18] C. Williamson, Vortex dynamics in the cylinder wake, Annu. Rev. Fluid Mech. 28, 477 (1996).

[19] U. Fey, M. König, and H. Eckelmann, A new Strouhal-Reynolds number relationship for the circular cylinder in the range $47<\operatorname{Re}<2 \times 10^{5}$, Phys. Fluids 10, 1547 (1998).

[20] M. O. Williams, I. G. Kevrekidis, and C. W. Rowley, A data-driven approximation of the koopman operator: Extending dynamic mode decomposition, J. Nonlin. Sci. 25, 1307 (2015).

[21] J. H. Tu, C. W. Rowley, D. M. Luchtenburg, S. L. Brunton, and J. N. Kutz, On dynamic mode decomposition: Theory and applications, J. Comput. Dynam. 1, 391 (2014).

[22] H. Arbabi and I. Mezić, Study of dynamics in post-transient flows using Koopman mode decomposition, Phys. Rev. Fluids 2, 124402 (2017).

[23] I. Mezić, Spectral properties of dynamical systems, model reduction, and decomposition, Nonlin. Dyn. 41, 309 (2005). 